\title{
When is the use of contrast media in chest CT indicated?
}

\author{
Bruno Hochhegger ${ }^{1,2,3}$, Robson Rottenfusser ${ }^{4,5}$, Edson Marchiori ${ }^{6}$
}

\section{TO THE EDITOR:}

It is undeniable that CT plays an important role in the diagnosis and treatment of various clinical conditions involving the chest wall, mediastinum, pleura, pulmonary arteries, and lung parenchyma. The need for enhancement using i.v. contrast media depends on the specific clinical indication. ${ }^{(1-3)}$ The most common contrast agents used with $\mathrm{CT}$ imaging are iodinated. Intravenous iodinated contrast agents are used for opacification of vascular structures. The major families of contrast agents are ionic and nonionic. Contrast agents can be further classified as high- or low-osmolality agents on the basis of the iodine concentration. Most centers use nonionic contrast agents (generally low-osmolality agents) for i.v. contrast studies. ${ }^{(1)}$ The rate of major reactions (e.g., anaphylaxis and death) is the same for i.v. ionic and nonionic contrast agents-an estimate of 1 in 170,000 administrations-but nonionic contrast agents show a lower rate of minor reactions. (2) Approximately 5-12\% of the patients who receive high-osmolality contrast media have adverse reactions, most of which are mild or moderate. ${ }^{(3-5)}$ The use of low-osmolality contrast agents has been associated with a reduction in adverse effects. Children have a lower incidence of reactions to i.v. contrast agents, and most of these are mild $(0.18 \%$ for low-osmolality agents). ${ }^{(3,4)}$ Risk factors for contrast agent reactions include multiple drug allergies and asthma. ${ }^{(5)}$ Although many radiology departments screen for shellfish allergy, there is no cross-reactivity between shellfish and iodinated contrast agents.

Actually, in the vast majority of cases requiring CT scans of the chest is not required the use of contrast media for an accurate diagnosis. The most prevalent indications for $\mathrm{CT}$, such as COPD, interstitial lung disease, pulmonary nodule, small or large airway disease, and lung cancer screening, do not require the use of any contrast media.

However, for the assessment of vascular disease, CT requires the use of an i.v. contrast agent to delineate the vessel lumen (e.g., aneurysm, dissection, and vascular tumor invasion). Pulmonary embolic disease is the third most common cause of acute cardiovascular disease..$^{(3,5)}$ Chest CT angiography is the most common way to assess for pulmonary embolic disease because, in addition to being accurate, fast, and widely available, it can assess alternate pathologies in cases of undifferentiated chest pain. Typically, the use of CT in order to investigate vascular disease or pleural pathology may include non-contrast-enhanced images to identify hemorrhage, enhancement, and calcifications. In addition, pleural enhancement is used in order to evaluate suspected or known exudative effusions and empyema. ${ }^{(2)}$ It also aids in evaluating metastatic or primary malignancy of the pleura, particularly in cases of occult disease, as enhancement and thickening of the pleura are of diagnostic interest.

The use of iodinated contrast agents in lung cancer is controversial. When there is suspected mediastinal invasion, the use of contrast media is indicated; however, other evaluations can be performed without it.

In conclusion, the use of contrast-enhanced chest CT imaging is indicated to investigate vascular and pleural diseases, but most of the scans can be obtained without contrast enhancement. Also, the specific agent and the route of administration are based on clinical indications and patient factors. Clear communication between the physician and the radiologist is essential for obtaining the most appropriate study at the lowest cost and minimum risk to the patient.

\section{REFERENCES}

1. Utter DP. Survey of contrast media use in southeastern U.S. hospitals. Radiol Technol. 1997;68(5):386-90. https://doi.org/10.1148/ radiology.175.3.2343107

2. Katayama H, Yamaguchi K, Kozuka T, Takashima T, Seez P, Matsuura K. Adverse reactions to ionic and nonionic contrast media. A report from the Japanese Committee on the Safety of Contrast Media. Radiology. 1990;175 (3):621-8

3. American College of Radiology. ACR manual on contrast media: version

8. Reston (VA): American College of Radiology; 2012.

4. Dillman JR, Strouse PJ, Ellis JH, Cohan RH, Jan SC. Incidence and severity of acute allergic-like reactions to i.v. nonionic iodinated contrast material in children. AJR Am J Roentgenol. 2007;188(6):1643-7. Erratum in: AJR Am J Roentgenol. 2007;189(3):512. https://doi.org/10.2214/AJR.06.1328

5. Coakley FV, Panicek DM. lodine allergy: an oyster without a pearl? AJR Am J Roentgenol. 1997;169(4):951-2. https://doi.org/10.2214/ ajr.169.4.9308442 